\title{
Nanoscale
}

A Check for updates

Cite this: Nanoscale, 2021, 13, 18160

\section{Role of topological surface states and mirror symmetry in topological crystalline insulator SnTe as an efficient electrocatalyst $\dagger$}

\author{
Qing Qu, (D) $\ddagger^{a, b}$ Bin Liu, (D) $\ddagger^{c}$ Hongtao Liu, (D) Jing Liang, c,b Jiannong Wang, (D) c,b \\ Ding Pan*c,d and lam Keong Sou*a,c,b
}

\begin{abstract}
The surface orientation dependence on the hydrogen evolution reaction (HER) performance of topological crystalline insulator (TCI) SnTe thin films is studied. Their intrinsic activities are determined by linear sweep voltammetry and cyclic voltammetry measurements. It is found that SnTe (001) and (111) surfaces exhibit intrinsic activities significantly larger than the (211) surface. Density functional theory calculations reveal that pure (001) and (111) surfaces are not good electrocatalysts, while those with Sn vacancies or partially oxidized surfaces, with the latter as evidenced by X-ray photoelectron spectroscopy, have high activity. The calculated overall performance of the (001) and (111) surfaces with robust topological surface states (TSSs) is better than that of the lowly symmetric (211) surface with fragile or without TSSs, which is further supported by their measured weak antilocalization strength. The high HER activity of SnTe (001) and (111) is attributed to the enhanced charge transfer between $\mathrm{H}$ atoms and TSSs. We also address the effect of possible surface facets and the contrast of the HER activity of the available active sites among the three samples. Our study demonstrates that the TSSs and mirror symmetry of TCls expedite their HER activity.
\end{abstract}

Received 4th August 2021

Accepted 27th September 2021

DOI: $10.1039 /$ d1nr05089c

rsc.li/nanoscale

In 2011, Fu extended the family of TIs by introducing topo-

\section{Introduction}

Recently, studies on a number of topological materials were reported both experimentally and theoretically to provide a novel direction for the design of high-efficiency non-noblemetal catalysts for the hydrogen evolution reaction (HER). ${ }^{1-5}$ It has been well accepted that the highly desirable functionality in topological insulator (TI)-based HER electrocatalysts is attributed to the topological surface states (TSSs) as revealed by first-principles density functional theory (DFT) calculations. ${ }^{4,6,7}$ In the past few years, several bismuth chalcogenide-based TIs have been proved to be promising candidates of photocatalysts, ${ }^{2}$ and $\mathrm{Bi}_{2} \mathrm{Te}_{3}$ thin films with partially oxidized structures or Te vacancies ${ }^{5}$ were shown to display high HER activities, providing evidence of the important role of TSSs in catalytic processes.

\footnotetext{
${ }^{a}$ Nano Science and Technology Program, The Hong Kong University of Science and Technology, Hong Kong, China. E-mail: phiksou@ust.hk

${ }^{b}$ William Mong Institute of Nano Science and Technology, The Hong Kong University of Science and Technology, Hong Kong, China

${ }^{c}$ Department of Physics, The Hong Kong University of Science and Technology,

Clear Water Bay, Hong Kong, China. E-mail: dingpan@ust.hk

${ }^{d}$ Department of Chemistry, The Hong Kong University of Science and Technology, Hong Kong, China

$\dagger$ Electronic supplementary information (ESI) available. See DOI: 10.1039/ d1nr05089c

$\$$ These authors contributed equally to this work.
}

logical crystalline insulators (TCIs) ${ }^{8,9}$ where the topology is protected by a point-group symmetry of the crystal lattice rather than by time reversal symmetry (TRS). The first theoretically predicted and experimentally realized TCI materials are IV-VI semiconductors, with SnTe as a representative. ${ }^{8-10}$ To the best of our knowledge, research on TCI thin film materials directly used as electrocatalysts in the HER with efficient performance has not been reported so far. It has been reported that there are two types of TCI surface states with qualitatively different electronic properties depending on the surface orientation: ${ }^{11}$ type-I TCI surface states on the (111) surface have the properties that their Dirac points are located at the time-reversal-invariant momenta (TRIMs), while the locations of Dirac points for type-II TCI surface states on the (001) and (110) surfaces are deviated from TRIMs. More importantly, the interplay between topology and crystal symmetry is the key characteristic of TCIs, which represents that if the mirror symmetry of SnTe is broken, the gapless surface states will be gapped. ${ }^{12}$

In this work, we prepared SnTe (111), SnTe (001) and SnTe (211) TCI thin films by molecular beam epitaxy (MBE) for studying the role of TSSs and mirror symmetry in electrocatalytic HER performance. SnTe (111), SnTe (001) and SnTe (211) were chosen as the representatives for type-I and type-II surfaces and a low-mirror-symmetric surface, respectively. It is revealed that the SnTe (001) and SnTe (111) samples exhibit lower overpotentials, Tafel slopes and charge-transfer resist- 
ances than those of the SnTe (211) sample. It was found that the turnover frequency (TOF) values of the SnTe (111) and SnTe (001) samples are comparable but much larger than that of the SnTe (211) sample. Our DFT calculations show that the Sn vacancy or the surface partial oxidation lifts the Dirac cones and promotes the charge transfer from the $\mathrm{H}$ atoms to the surfaces, which makes the $\mathrm{H}$ adsorption easier, so the HER performance is enhanced. The findings from the X-ray photoelectron spectroscopy (XPS) and magneto-transport measurements performed on these samples are consistent with the theoretical calculations.

\section{Results}

A seemingly straightforward approach to fabricate three SnTe thin films with different orientations required by this study is to grow SnTe (111), SnTe (001) and SnTe (211) directly on GaAs (111)B, GaAs (001) and GaAs (211)B substrates. However, this approach was found to be unachievable for the reasons to be addressed below.

The high-resolution X-ray diffraction (HRXRD) profile of a SnTe thin film directly grown on a GaAs (111)B substrate is shown in Fig. S1 in the ESI, $\dagger$ where one can see that the resulting SnTe thin film is oriented along the [001] direction. Taskin et al. have previously demonstrated that SnTe (111) can be grown directly on the $\mathrm{Bi}_{2} \mathrm{Te}_{3}$ (001) surface, ${ }^{13}$ which was attributed to the small lattice mismatch of $1.5 \%$ between these two surfaces and the similarity of their surface unit cells. In the current study, in order to avoid the effects of the TSSs of $\mathrm{Bi}_{2} \mathrm{Te}_{3}$, we have adopted the following approach for fabricating a SnTe (111) layer. A ZnSe (111) buffer layer was first deposited on a GaAs (111)B substrate followed by the growth of a $\mathrm{Bi}_{2} \mathrm{Te}_{3}$ : $\mathrm{Fe}$ (001) layer. Our previous study reveals that light doping of Fe in $\mathrm{Bi}_{2} \mathrm{Te}_{3}$ can destroy the TSSs of $\mathrm{Bi}_{2} \mathrm{Te}_{3}$ as supported by the quenching of the weak antilocalization (WAL) signature. ${ }^{5}$ The growth of the top SnTe (111) layer was then followed to complete the growth of this sample.

On the other hand, it was found that SnTe (001) thin film can be directly grown on a GaAs (001) substrate. For the growth of the SnTe (211) thin film, since the GaAs (211)B substrate is not an epi-ready substrate, the required wet chemical etching process was found to generate a rather rough surface, thus a thick ZnSe buffer layer was first grown prior to the growth of the top SnTe (211) layer.

\section{Structural characterization of MBE-grown SnTe (111), (001) and (211) samples}

Fig. S2 in the ESI $\dagger$ shows the sample structures of the MBEgrown SnTe (111), SnTe (001), and SnTe (211) samples and the reflection high-energy electron diffraction (RHEED) patterns captured during the growth. The streaky RHEED patterns of the surfaces of $\mathrm{Bi}_{2} \mathrm{Te}_{3}: \mathrm{Fe}(0001)$, SnTe (111) and SnTe (001) indicate their single crystalline nature. A video of the RHEED patterns of the SnTe (211) surface is provided in the ESI $\dagger$ to show its single crystalline nature. The details of the RHEED studies on the three SnTe samples are addressed in the remarks of Fig. S2. $\dagger$

Fig. 1a-c display the HRXRD profiles of the SnTe (111), SnTe (001), and SnTe (211) samples. Fig. 1d-g show the powder diffraction files (PDFs) of the four crystalline materials contained in these samples, where only the peaks oriented along the normal of the sample surface are extracted for comparison. Fig. 1a displays the HRXRD profile of the SnTe (111) sample. As can be seen, all $\mathrm{Bi}_{2} \mathrm{Te}_{3}$ layer peaks can be indexed to the $(00 l)$ direction and their measured $2 \theta$ values give a $c$-lattice parameter of $\sim 30.478 \AA$, which closely matches the PDF values of $\mathrm{Bi}_{2} \mathrm{Te}_{3}(c=30.483 \AA)$. The characteristic diffraction peak located at $50.04^{\circ}$ matches well with the standard $2 \theta$ value of SnTe (222) giving a lattice constant of $6.309 \AA$, which has a deviation of only $0.29 \%$ from the standard value of $6.33 \AA$ A. The diffraction peaks of ZnSe (111) and ZnSe (222) overlap with the strong peaks of GaAs (111) and GaAs (222) in this broad-scan profile due to the very small lattice mismatch $(0.27 \%)$ between ZnSe and GaAs. ${ }^{5,14,15}$ Fig. 1b displays the HRXRD profile of the SnTe (001) sample. Two characteristic diffraction peaks located at $28.235^{\circ}$ and $58.365^{\circ}$ match with the standard $2 \theta$ values of SnTe (002) and (004), respectively. From these two peaks, the lattice constant of the SnTe (001) sample is determined to be $6.32 \AA$, which agrees well with the standard value from the PDF (6.33 $\AA$ ), indicating that the SnTe

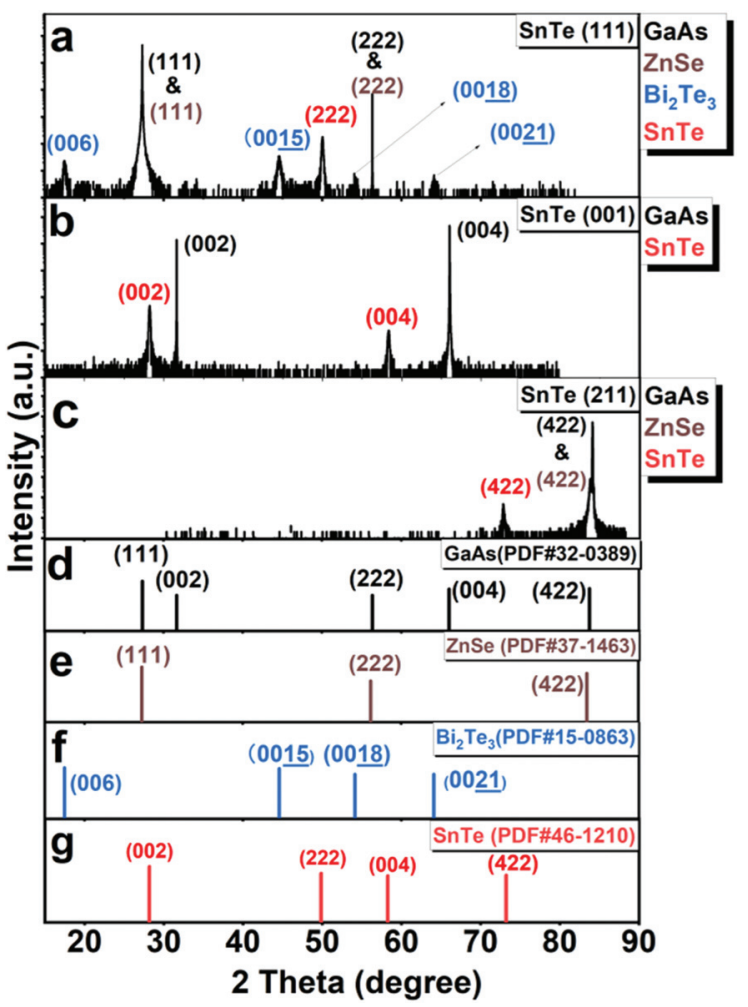

Fig. 1 High-resolution X-ray diffraction (HRXRD) $2 \theta-\omega$ scan profiles of the three MBE-grown thin film samples and the corresponding powder diffraction files (PDFs). (a) SnTe (111), (b) SnTe (001) and (c) SnTe (211) samples. (d-g) PDFs for the four materials contained in the three thin film samples. 
(001) layer is almost fully relaxed. Fig. 1c shows the HRXRD profile of the SnTe (211) sample. The characteristic diffraction peak located at $72.55^{\circ}$ matches with the standard $2 \theta$ values of SnTe (422) giving the lattice constant to be $6.378 \AA$, which is also close to the standard value of $6.33 \AA$ A. The cross-sectional high-resolution transmission electron microscopy (HRTEM) images with fast Fourier transform (FFT) images and corresponding atomic arrangements of the three SnTe samples studied in this work are shown in Fig. S3, $\uparrow$ which further support the findings from HRXRD. The details of the HRTEM studies on the three SnTe samples are addressed in the remarks of Fig. S3. $\dagger$ Fig. $\mathrm{S} 4 \uparrow$ shows the top-view atomic match correlation between the two domains of the $\mathrm{Bi}_{2} \mathrm{Te}_{3}$ : $\mathrm{Fe}(001)$ layer and the SnTe (111) layer, together with the distances between two neighbouring atoms of $\mathrm{Te}$ in the $\mathrm{Bi}_{2} \mathrm{Te}_{3}: \mathrm{Fe}(001)$ lattice and $\mathrm{Sn}$ in the SnTe (111) lattice. One can see that these two layers have similar in-plane lattice arrangement with only $1.59 \%$ in lattice mismatch, which explains why the SnTe (111) layer can be epitaxially grown on top of the $\mathrm{Bi}_{2} \mathrm{Te}_{3}: \mathrm{Fe}(001)$ layer.

Atomic force microscopy (AFM) images of the surfaces and the profile analyses of the three SnTe samples are shown in Fig. 2. Fig. 2a-c show the AFM images of the surface of the SnTe (111) sample with increasing magnification. Fig. 2d shows the profile analyses along the green and blue lines in Fig. 2 c for 5 different positions. Triangular domains and ter- races are clearly seen on this surface as shown in Fig. 2a-c. As shown in the profile analyses in Fig. 2d, for the layers forming the terraces, their heights are found to be either around $1 \mathrm{~nm}$ or $0.37 \mathrm{~nm}$, which corresponds to the thickness of a single quintuple layer of $\mathrm{Bi}_{2} \mathrm{Te}_{3}$ or the step height of one SnTe (111) bilayer (BL) $(0.365 \mathrm{~nm}) .{ }^{16}$ The terraces with a step height of $1 \mathrm{~nm}$ provide the evidence that the SnTe (111) layer follows the morphology of the triangular domains of the $\mathrm{Bi}_{2} \mathrm{Te}_{3}: \mathrm{Fe}(001)$ surface, while the terraces with step height around $0.37 \mathrm{~nm}$ indicate that the growth of SnTe (111) also occurs in a BL-byBL manner. Fig. 2e-g show the AFM images of the surface of the SnTe (001) sample with increasing magnification. Fig. $2 \mathrm{~h}$ shows the profile analyses along the green and blue lines. It can be seen that the surface of SnTe (001) exhibits a valley structure with holes and ditches. Such a surface morphology is believed to result from an initial island growth mode followed by the coalescence of some preferred islands into continuous stripes, similar to the observations by Ishikawa et al. ${ }^{17}$ The profile analyses carried out for some typical holes and ditches are shown in Fig. 2h. It was found that the diameters of the holes and the widths of the ditches have sizes ranging from 120 to $210 \mathrm{~nm}$, and the depths of the holes and the ditches range from 5 to $25 \mathrm{~nm}$. Fig. 2i-k show the AFM images of the surface of the SnTe (211) sample with increasing magnification, which shows that the surface morphology of this sample is dominated by coalescing islands. Fig. 21 shows the
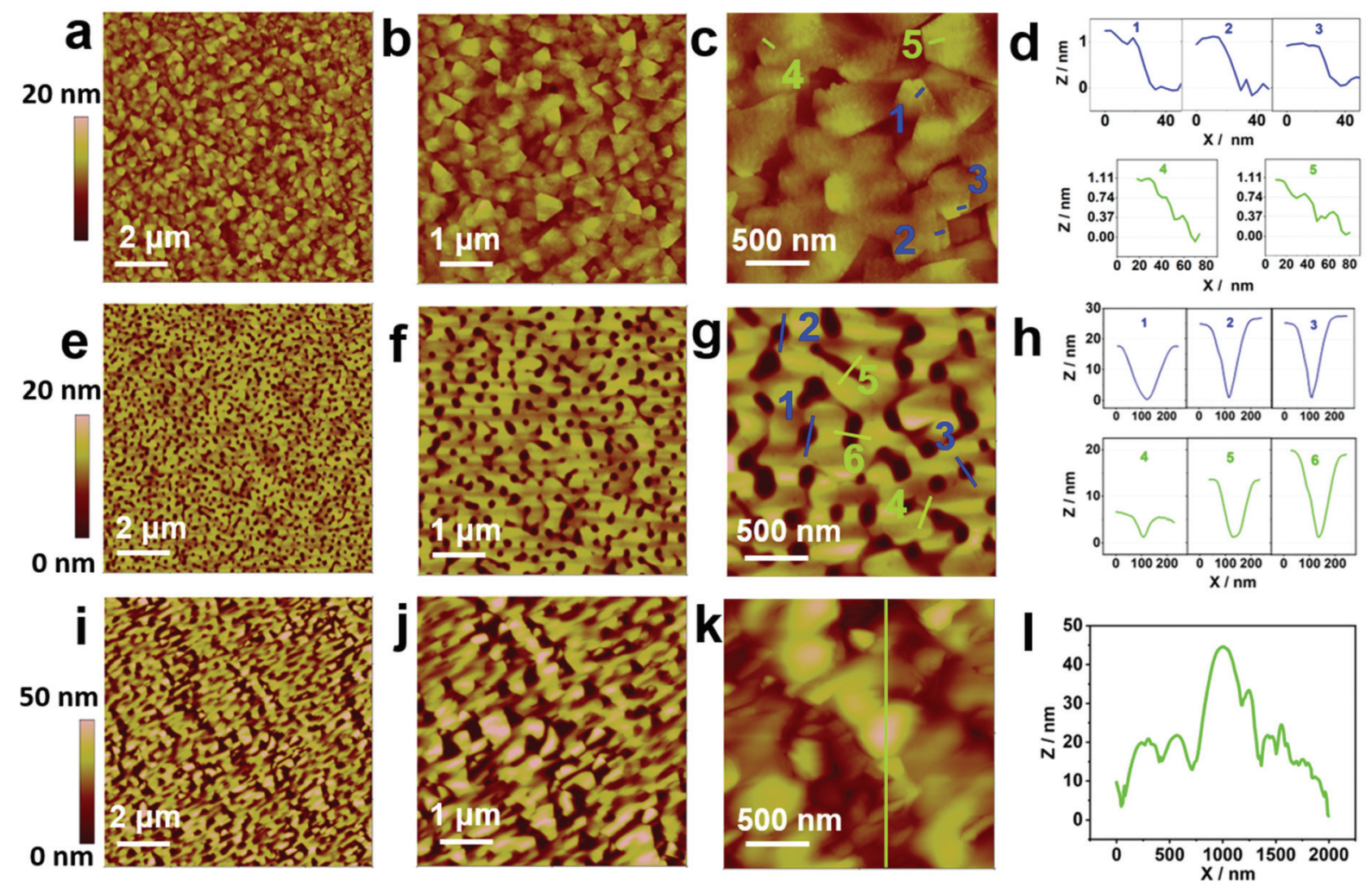

Fig. 2 Morphology analysis of the three SnTe samples. Atomic force microscopy (AFM) images of the MBE-grown (a-c) SnTe (111), (e-g) SnTe (001), and (i-k) SnTe (211) samples. The profile analyses of ( $c, g$ and $k$ ) along the green and blue lines are presented in (d, h and $l)$ respectively. 
profile analysis along the green line in Fig. $2 \mathrm{k}$, indicating that the surface roughness can reach as high as $50 \mathrm{~nm}$.

\section{Electrocatalytic HER performance of the SnTe (001), (111) and (211) samples}

We have examined the electrochemical catalytic HER behaviors of the as-grown SnTe (001), (111), and (211) samples and a commercial Pt foil for comparison, and the results are presented in Fig. 3. Fig. 3a displays the linear sweep voltammogram (LSV) polarization curves of these materials, and as shown in Table 1, the overpotentials $(\eta)$ of the SnTe (001), SnTe (111) and SnTe (211) samples at a cathodic current density $(j)$ of $10 \mathrm{~mA} \mathrm{~cm}{ }^{-2}$ are $198 \mathrm{mV}, 307 \mathrm{mV}$, and $366 \mathrm{mV}$, respectively. The SnTe (001) thin film exhibits the lowest $\eta$ among them, even lower than the value of $48 \mathrm{~nm}$ for the $\mathrm{Bi}_{2} \mathrm{Te}_{3}$ thin film reported previously by our group; ${ }^{5}$ the latter is among the best values of HER performances when compared with reported TI materials (exfoliated $\mathrm{Bi}_{2} \mathrm{Te}_{3}, \mathrm{Bi}_{2} \mathrm{Se}_{3}, \mathrm{Sb}_{2} \mathrm{Te}_{3}, \mathrm{Bi}_{0.5} \mathrm{Sb}_{1.5} \mathrm{Te}_{3}$ alloy), and some molybdenum dichalcogenide $\left(\mathrm{MoX}_{2}\right)$ based nanosheet catalysts.

The kinetics of active catalytic materials during the HER process can be revealed by the Tafel slope. ${ }^{18}$ For the HER in acidic media, two different mechanisms, Volmer-Tafel and
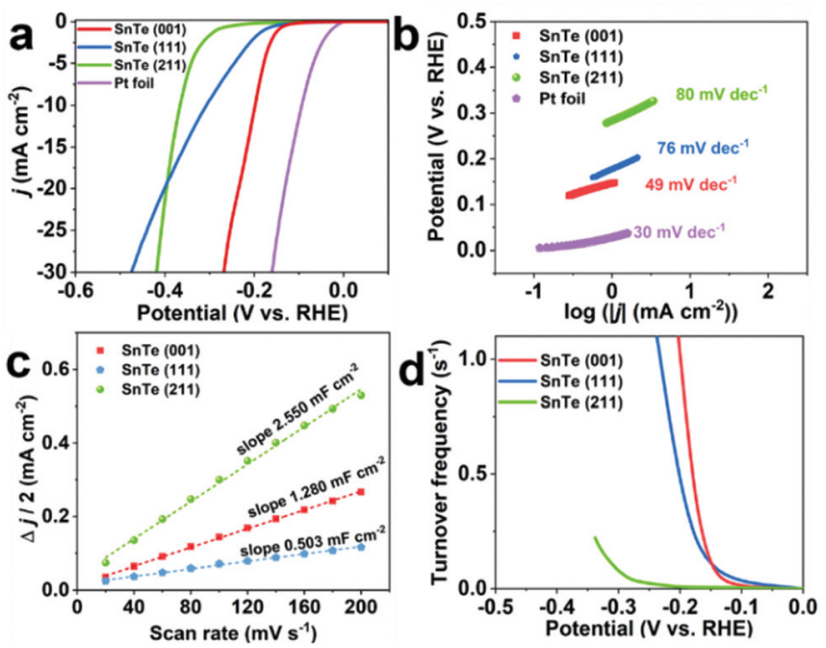

Fig. 3 HER electrocatalytic performances of the SnTe (001), SnTe (111) and SnTe (211) samples. (a) Polarization curves (iR-corrected) of the three SnTe samples and a commercial Pt foil. (b) Corresponding Tafel plots of the materials in (a). (c) Linear fits of the half capacitive currents as a function of scan rates for the extraction of $C_{\mathrm{dl}}$ values of the three SnTe samples. (d) Turnover frequency (TOF) plots against the potential for the three SnTe samples.
Volmer-Heyrovsky, have been recognized to be responsible for transforming $\mathrm{H}^{+}$to $\mathrm{H}_{2}$, which include three principal steps:

$\mathrm{H}^{+}+\mathrm{e}^{-}+{ }^{*} \rightarrow \mathrm{H}^{*}$

(Volmer reaction : electrochemical hydrogen adsorption)

$\mathrm{H}^{*}+\mathrm{H}^{+}+\mathrm{e}^{-} \rightarrow \mathrm{H}_{2}+{ }^{*}$

(Heyrovsky reaction : electrochemical desorption)

$2 \mathrm{H} * \rightarrow \mathrm{H}_{2}+2 * \quad$ (Tafel reaction : chemical desorption)

where ${ }^{*}$ denotes a site on the electrode surface. ${ }^{19}$ The hydrogen evolution pathway and the rate-determining step can be deduced from the value of the Tafel slope, which is about 120, 40 , or $30 \mathrm{mV} \mathrm{dec}{ }^{-1}$ if the Volmer, Heyrovsky, or Tafel step is the rate-determining step, respectively. ${ }^{18}$ The corresponding Tafel plots of the active materials shown in Fig. 3a are displayed in Fig. $3 \mathrm{~b}$. The linear portions of the plots shown in Fig. $3 \mathrm{~b}$ are fitted to the Tafel equation $(\eta=b \log j+a$, where $j$ is the current density and $b$ is the Tafel slope; the fitting equations are displayed in Fig. S6†). As shown in Fig. 3b, the Tafel slope of the Pt foil as a reference is $30 \mathrm{mV} \mathrm{dec}^{-1}$ which is consistent with the expected value of the known HER mechanism on Pt, in which the HER occurs through a Volmer-Tafel mechanism and the overall reaction is determined by the Tafel step. As shown in Fig. 3b and Table 1, for the SnTe (001), SnTe (111) and SnTe (211) samples, the respective Tafel slopes of $49 \mathrm{mV} \mathrm{dec}^{-1}, 76 \mathrm{mV} \mathrm{dec}{ }^{-1}$ and $80 \mathrm{mV} \mathrm{dec}{ }^{-1}$ suggest a VolmerHeyrovsky mechanism, despite different rate-determining steps. For the SnTe (111) and SnTe (211) samples, the overall HER rate is determined by a mixed Volmer-Heyrovsky step due to the Tafel slopes relatively close to the average value of that of these two steps. ${ }^{20-22}$ The fact that the SnTe (001) sample has a lower Tafel slope of $49 \mathrm{mV} \mathrm{dec}{ }^{-1}$ implies that its HER process is determined mainly by a typical Heyrovsky step rather than a coupled Volmer-Heyrovsky step. ${ }^{22,23}$ This value is close to that of $\mathrm{Bi}_{2} \mathrm{Te}_{3}(48 \mathrm{~nm})\left(47.87 \mathrm{mV} \mathrm{dec}^{-1}\right)$ we reported previously, which has already been among the best values of the other TI materials and some $\mathrm{MoX}_{2}$-based catalysts. The Tafel slope of the SnTe (001) sample is the lowest among the three SnTe samples, which is consistent with the LSV results shown in Fig. 3a. Furthermore, the exchange current densities $\left(j_{0}\right)$ were obtained by extrapolating the x-intercepts $(\eta=0)$ of the fitted dash lines shown in Fig. S6. $\dagger$ The obtained values of $j_{0}$ and the corresponding $\log j_{0}\left(\mathrm{~A} \mathrm{~cm}^{-2}\right)$ which is an important parameter for constructing the volcano $\operatorname{plot}^{19}$ are shown in Table 1 . The $\log j_{0}$ values $\left(\mathrm{A} \mathrm{cm}^{-2}\right)$ of the SnTe (001) sample

Table 1 Comparison of the HER performance of the three SnTe (111), SnTe (001) and SnTe (211) samples

\begin{tabular}{|c|c|c|c|c|c|c|}
\hline Samples & $\begin{array}{l}\text { Overpotential } \\
(\mathrm{mV} v s . \mathrm{RHE})\end{array}$ & $\begin{array}{l}\text { Tafel slope } \\
\left(\mathrm{mV} \mathrm{dec}{ }^{-1}\right)\end{array}$ & $\begin{array}{l}\text { Exchange current } \\
\text { density }\left(j_{0}\right)\left(\mu \mathrm{A} \mathrm{cm}^{-2}\right)\end{array}$ & $\begin{array}{l}\log j_{0} \\
\left(\mathrm{~A} \mathrm{~cm}{ }^{-2}\right)\end{array}$ & $R_{\mathrm{ct}}(\Omega)$ & $\begin{array}{l}\text { TOF at } \\
200 \mathrm{mV}\left(\mathrm{s}^{-1}\right)\end{array}$ \\
\hline SnTe (001) & 198 & 49 & 1.065 & -5.973 & 19.84 & 1.034 \\
\hline SnTe (111) & 307 & 76 & 4.592 & -5.338 & 23.38 & 0.482 \\
\hline SnTe (211) & 366 & 80 & 0.288 & -6.541 & 44.50 & 0.009 \\
\hline
\end{tabular}


$(-5.973)$ and the SnTe (111) sample $(-5.338)$ are in the same order, while the $\log j_{0}\left(\mathrm{~A} \mathrm{~cm}^{-2}\right)$ of the SnTe (211) sample $(-6.541)$ is significantly lower.

To gain a better understanding of the interface reactions and electrode kinetics mechanism, electrochemical impedance spectroscopy (EIS) measurements were also performed. The Nyquist plots of the SnTe (001), SnTe (111) and SnTe (211) samples are given in Fig. S7. $\dagger$ We can derive their charge transfer resistance $\left(R_{\mathrm{ct}}\right)$ by applying a simplified Randles circuit model as shown in the inset of Fig. S7. $\dagger$ The resulting $R_{\mathrm{ct}}$ values are shown in Table 1 in which one can see that the SnTe (001) sample exhibits the lowest $R_{\text {ct }}$ value of $19.84 \Omega$, while that of the SnTe (111) sample and the SnTe (211) sample is $23.38 \Omega$ and $44.50 \Omega$, respectively, which is consistent with the observation that among the three SnTe samples, the values of $\eta$ and Tafel slope of the SnTe (211) sample are the highest and $j_{0}$ is the lowest. We believe that the higher performance in the HER (represented by the values of $\eta$ and Tafel slope) of the SnTe (001) sample over that of the SnTe (111) sample may be partially attributed to the valley surface structures with holes and ditches of the former sample as shown in the AFM images in Fig. 2, which offers more active areas. However, the SnTe (211) sample exhibits the roughest surface morphology (as shown in Fig. 2) but the worst electrocatalytic activity among the three SnTe samples. Thus, further HER characteristic analysis about their intrinsic electrocatalytic activity is thus essential, which has been studied and the results will be addressed below.

It is well known that the intrinsic electrocatalytic activity of a catalytic surface can be evaluated by extracting the TOF, which requires one to determine the ratio between the number of total hydrogen turnover per $\mathrm{cm}^{2}$ and the number of active sites per $\mathrm{cm}^{2} \cdot{ }^{24}$ A detailed description of this method and our calculations are provided in ESI note $1 . \dagger$ Fig. $3 \mathrm{c}$ shows the plots of $\Delta j / 2$ versus scan rate of the three SnTe samples, in which the slopes representing the double-layer capacitance $\left(C_{\mathrm{dl}}\right)$ were extracted to be $2.550 \mathrm{mF} \mathrm{cm}^{-2}, 1.280 \mathrm{mF} \mathrm{cm}^{-2}$ and $0.503 \mathrm{mF} \mathrm{cm}^{-2}$ for the SnTe (211), (001) and (111) samples, respectively. The electrochemically active surface areas (ECSAs) were estimated from the $C_{\mathrm{dl}}$ values as shown in ESI note $1 . \dagger$ The estimated ECSA values are consistent with the AFM image analysis as shown in Fig. 2 regarding the degree of surface roughness. Fig. 3d shows the TOF values versus the potentials for the three SnTe samples. Apparently, the TOF values of the SnTe (001) and SnTe (111) samples are comparable but much larger than that of the SnTe (211) sample.

As an example, the long-term stability of the SnTe (001) sample as an HER electrocatalyst was evaluated via the cycling stability test conducted at a scan rate of $100 \mathrm{mV} \mathrm{s}^{-1}$. Fig. S9† shows the polarization curves recorded before and after 1000 cycles of cyclic voltammetry (CV) treatments. As can be seen in Fig. S9, $\dagger$ there is an observed potential increase of only $18.9 \mathrm{mV}$ at a cathodic current density $j=10 \mathrm{~mA} \mathrm{~cm}{ }^{-2}$ after the $\mathrm{CV}$ treatments, representing that the electrochemical HER process does not cause significant loss in the electrocatalytic performance of the SnTe (001) sample. This slight degradation may arise from the fact that some active components may be exfoliated during the $\mathrm{CV}$ treatments due to the disturbance from the evolution of $\mathrm{H}_{2}$ bubbles generated on the SnTe (001) thin-film cathode. ${ }^{25}$

\section{First-principles calculations}

To understand the mechanism of the high catalytic efficiency induced by metallic TSSs on SnTe surfaces, we applied the DFT method to calculate the free energy of hydrogen adsorption $\left(\Delta G_{\mathrm{H}}\right)$ (see Methods), ${ }^{19,26,27}$ which is a good descriptor for the performance of HER catalysts. Either strong binding $\left(\Delta G_{\mathrm{H}}\right.$ $\ll 0 \mathrm{eV})$ or strong repulsion $\left(\Delta G_{\mathrm{H}} \gg 0 \mathrm{eV}\right)$ hurts the catalytic performance. The optimal value for $\Delta G_{\mathrm{H}}$ is close to $0 \mathrm{eV}$. For example, $\Delta G_{\mathrm{H}}$ of Pt is $-0.18 \mathrm{eV}$ as calculated by the PerdewBurke-Ernzerhof (PBE) exchange-correlation functional when the hydrogen coverage on the (111) surface is $1 / 4$ monolayer (ML). ${ }^{28,29}$

Fig. 4 shows the $\Delta G_{\mathrm{H}}$ values for pure, Sn-vacancy-containing, and partially oxidized surfaces of SnTe. We considered the effects of hydrogen coverage, Sn vacancy, and oxygen concentrations by varying the size of unit cells and the number of oxygen atoms. In the following, we mainly compare the $\Delta G_{\mathrm{H}}$ values when the hydrogen coverage is $1 / 4 \mathrm{ML}$. On either the (001) or Te-terminated (111) surface, $\Delta G_{\mathrm{H}}$ is $0.60 \mathrm{eV}$, which indicates that the pure SnTe surfaces strongly repel the $\mathrm{H}$ atom and they do not work well for the HER (we discussed the Snterminated (111) surface in ESI note $2 \dagger$ ). In fact, previous studies suggested that the experimental SnTe films were not perfect, but might contain many $\mathrm{Sn}$ vacancies ${ }^{10,30}$ and were also partially oxidized. ${ }^{31,32}$ After introducing the $\mathrm{Sn}$ vacancy, $\Delta G_{\mathrm{H}}$ decreases considerably to $0.04 \mathrm{eV}$ for the (001) surface and $0.01 \mathrm{eV}$ for the (111) surface, both of which are closer to zero than $\Delta G_{\mathrm{H}}$ for the (211) surface with the Sn vacancy $(0.12 \mathrm{eV})$. Partial oxidation of SnTe surfaces may also provide extra active sites for $\mathrm{H}$ adsorption. As shown in Fig. $4, \Delta G_{\mathrm{H}}$ for the partially oxidized (001) surface is reduced to $-0.11 \mathrm{eV}$,

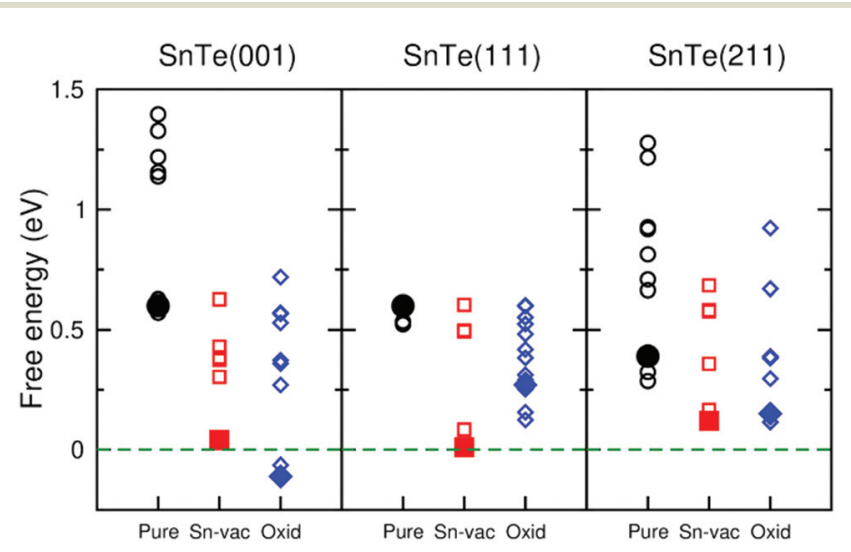

Fig. 4 Free energies of hydrogen adsorption $\left(\Delta G_{H}\right)$ on the SnTe (001), (111), and (211) slabs. There are three types of surfaces: pure (black circles), Sn-vacancy-containing (red squares), and partially oxidized (blue diamonds). The hydrogen coverage is between 1/9 ML and $1 \mathrm{ML}$. The filled symbols show the lowest $\left(\Delta G_{H}\right)$ with hydrogen coverage of $1 /$ $4 \mathrm{ML}$. The atomic structures are shown in Fig. S10-S18. $\dagger$ 
much closer to zero than $\Delta G_{\mathrm{H}}$ for the partially oxidized (211) surface $(0.15 \mathrm{eV})$. Partial oxidation on the (111) surface also reduces $\Delta G_{\mathrm{H}}$ to $0.27 \mathrm{eV}$. If the partial oxidation of a SnTe surface dominates over Sn vacancies and the surface roughness factor is not taken into account, it is thus expected that the SnTe (001) surface may offer a higher HER performance than the SnTe (111) surface. Overall, our DFT calculations show that the Sn vacancy and partial oxidation considerably decrease $\Delta G_{\mathrm{H}}$, and thus enhance the electrocatalytic performance of SnTe. The decrease of $\Delta G_{\mathrm{H}}$ is more apparent for the (001) and (111) surfaces than for the (211) surface.

We performed electronic structure calculations to understand the enhancement of the electrocatalytic performances of SnTe. Fig. 5 shows that the Dirac cone shifts to a higher energy level after introducing an Sn vacancy site on the (001) surface compared to the pure surface, which makes SnTe a p-type material. ${ }^{10,33}$ Consequently, the projected density of states (PDOS) on either Sn or Te near the Fermi level largely increases (Fig. S19†), which prompts the chemical bonds between the $\mathrm{H}$ atom and the Sn-vacancy-containing surfaces, and thus $\Delta G_{\mathrm{H}}$ decreases considerably as compared with the pure surface

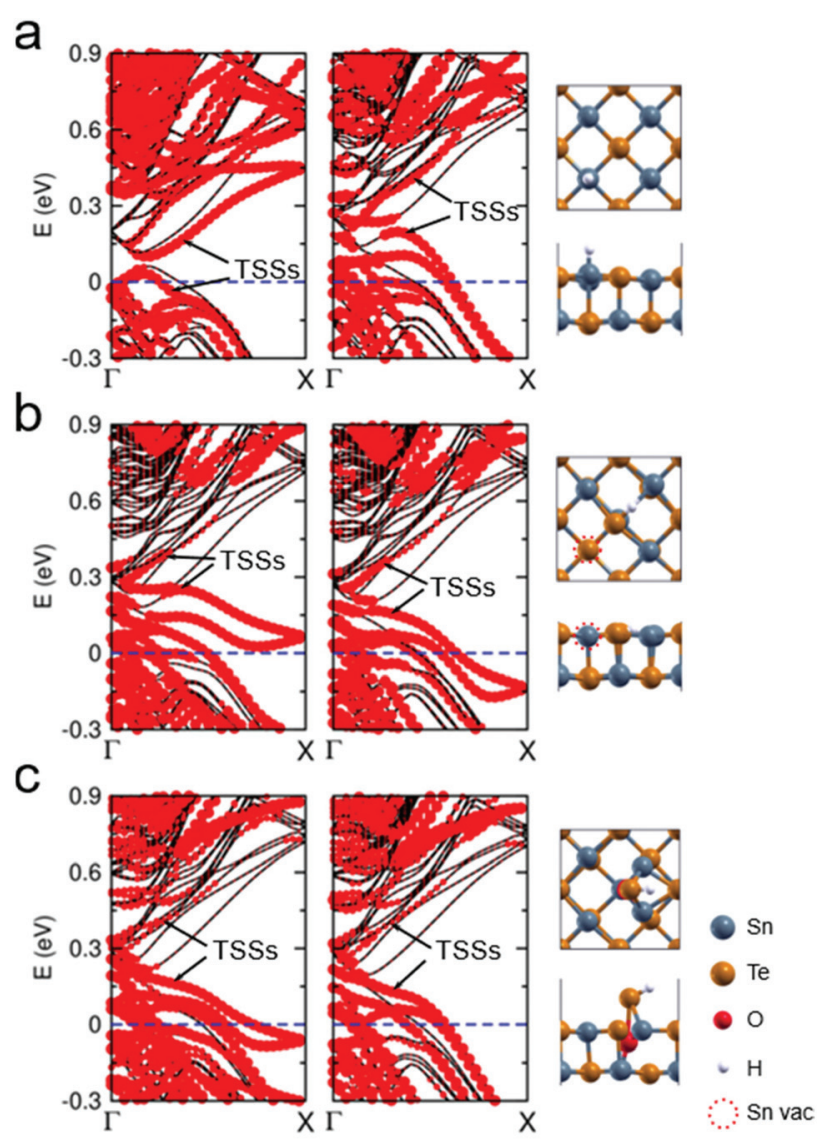

Fig. 5 Band structures of various SnTe basal slabs before (left panel) and after (middle panel) hydrogen adsorption, and the top and side views of the surface slabs (right panel). (a) Pure, (b) Sn-vacancy-containing, and (c) partially oxidized surfaces of the SnTe (001) $2 \times 2$ slab. The sizes of red dots represent the contributions from the (001) upper surface. The Fermi levels of the slabs are set to zero. adsorption (Table $\mathrm{S} 2 \dagger$ ). When the $\mathrm{H}$ atom is adsorbed on the pure surfaces, the Dirac cone upshifts due to the electron transfer from TSSs to the adsorbed $\mathrm{H}$ atom (see Fig. 5a and S20a†), which does not favor a stable chemical bond. As a comparison, the $\mathrm{H}$ adsorption on the Sn-vacancy-containing surfaces makes the Dirac cone downshift slightly (see Fig. 5b and S20 $\mathrm{b}_{\dagger}$ ), consistent with the bond formation between the $\mathrm{H}$ atom and the surfaces. The Löwdin population analysis ${ }^{34}$ (Table S3†) also shows that the charge is transferred from the $\mathrm{H}$ atom to the (001) surface, so the $\mathrm{H}$ atom becomes easier to attach the Sn-vacancy surface as compared with the pure case. For the (111) surface, the Dirac cone has a similar upshift after introducing the Sn vacancy, ${ }^{35}$ leading to a significant increase of PDOS on Te atoms near the Fermi level, which also makes the $\mathrm{H}$ adsorption easier (Fig. S21a and $\mathrm{b} \dagger$ ). As a comparison, the (211) surface without TSSs does not have the Dirac cone, so the decrease of $\Delta G_{\mathrm{H}}$ is mainly due to dangling bond states near the Fermi level, which we will discuss later.

Our electronic structure calculations show that the surface oxidation plays a similar role to the $\mathrm{Sn}$ vacancy in the $\mathrm{H}$ adsorption. Fig. $5 \mathrm{c}$ shows that the Dirac cone moves to a higher energy level on the partially oxidized (001) surface and downshifts slightly after the $\mathrm{H}$ adsorption. As shown in Fig. S20c, $\uparrow$ the PDOS on Te atoms increases due to the upshift of the Dirac cone (Fig. S19a $\dagger$ ), which favors the electron transfer from the $\mathrm{H}$ atom to the (001) surface (Table $\mathrm{S} 3 \dagger$ ) and leads to the formation of a $\mathrm{H}$-Te bonding state below the Fermi level. Thus, $\Delta G_{\mathrm{H}}$ on the partially oxidized (001) surface decreases to $-0.11 \mathrm{eV}$, as shown in Fig. 4. A similar enhancement of the HER efficiency was also expected on the partially oxidized (111) surface (see Fig. S21c $\dagger$ ), where $\Delta G_{\mathrm{H}}$ decreases from 0.60 to $0.27 \mathrm{eV}$ as shown in Fig. 4. In our previous work, we also found that the partial oxidation on the $\mathrm{TI} \mathrm{Bi}_{2} \mathrm{Te}_{3}$ helps to improve the HER activity. ${ }^{5}$ As a comparison, we also studied the partial oxidation on the (211) surface without TSSs, and found that $\Delta G_{\mathrm{H}}$ decreases from $0.39 \mathrm{eV}$ to $0.15 \mathrm{eV}$, which is due to dangling bond states as mentioned earlier.

Vacancies or surface oxidation may provide dangling bond states, which may influence the electrocatalytic activity. ${ }^{5,26,36-43}$ To compare the effects of TSSs and dangling bond states on the catalytic activities of SnTe films, we calculated the electronic structure of the pure Te-terminated SnTe (111) slab. In Fig. S22a and $\mathrm{c}, \dagger$ the electronic states from unsaturated Te atoms at the (111) upper surface appear at the Fermi level, and after the $\mathrm{H}$ adsorption they disappear and the Dirac cones recover at the $\Gamma$ and $M$ points (Fig. S22b and $\mathrm{c} \dagger$ ). The Te-terminated SnTe (111) slab still has a large $\Delta G_{\mathrm{H}}$ value $(0.60 \mathrm{eV})$, indicating that the dangling bond states cannot enhance the HER performance much. After introducing the Sn vacancy underneath the Te-terminated (111) surface, the Dirac cone moves up and promotes the electron transfer between the $\mathrm{H}$ atom and the surface, leading to the formation of the $\mathrm{H}-\mathrm{Te}$ bond, which does not happen to the pure (111) surface (Fig. S21a and $\mathrm{b}_{\dagger}$ ) (note: on the pure (111) surface, there is no stable chemical bond, because the binding energy is positive. The peak in PDOS may come from some orbital overlaps, but 
does not mean any bond). Additionally, we also examined the effect of dangling bond states of the (211) surface. The dangling bond states of the (211) surface appear near the Fermi level after including the Sn vacancy (Fig. S23a and $\mathrm{b} \dagger$ ), and $\Delta G_{\mathrm{H}}$ decreases from $0.39 \mathrm{eV}$ to $0.12 \mathrm{eV}$, indicating that the dangling bond states may promote HER activity. However, the decrease of the $\Delta G_{\mathrm{H}}$ value (by $0.27 \mathrm{eV}$ ) caused by the $\mathrm{Sn}$ vacancy on the (211) surface is smaller than that caused by the Sn vacancy on the (001) or (111) surfaces (0.56 or $0.59 \mathrm{eV})$, implying that metallic TSSs play a major role in the enhancement of the HER activity.

Let us summarize our DFT calculation results. We found that the pure SnTe (001) and (111) surfaces do not favor the $\mathrm{H}$ adsorption, indicating that they are not good HER electrocatalysts. The Sn vacancy or the partial oxidation in the surfaces lifts the Dirac cones and facilitates the charge transfer between $\mathrm{H}$ atoms and the TSSs during the adsorption process, which boosts the HER performance. The overall performance of the (001) and (111) surfaces with TSSs is better than that of the (211) surface without TSSs, which indicates that TSSs are of great importance in the enhancement of the HER activity, consistent with our experimental results.

\section{XPS studies of the SnTe (111), (001) and (211) samples}

The XPS technique is a well-known tool for studying the chemical states of the surface elements of a sample. Since our theoretical studies revealed that the efficient electrocatalytic performance of the SnTe (001) sample may come from the specific dilute oxidized structures, we have performed XPS depth-profiling on the SnTe (001) sample to study the composition of the oxides near the surface. Fig. S28t shows the obtained XPS spectra near the Te 3d (Fig. S28a $\dagger$ ) and Sn 3d (Fig. S28b $\dagger$ ) core levels of the fresh surface and those after sputtering $30 \mathrm{~s}, 60 \mathrm{~s}$ and $90 \mathrm{~s}$ for this sample. As can be seen in the top spectra in Fig. S28a, $\uparrow$ which are obtained before $\mathrm{Ar}^{+}$ sputtering, a peak appears on the left-hand side of the two Te core levels. These two peaks can be assigned to $\mathrm{Te}$ in $\mathrm{TeO}_{2}$ because their binding energies are close to the reported standard values of $576.4 \mathrm{eV}\left(\mathrm{Te} 3 \mathrm{~d}_{5 / 2}\right)$ and $586.8\left(\right.$ Te $\left.3 \mathrm{~d}_{3 / 2}\right)$ of this oxide. $^{44,45}$ It can also be seen that the intensities of the two major peaks in Fig. S28a† increase while their full width at half maxima (FWHM) get narrower with the sputter time. As shown in Fig. S28b, $\uparrow$ the value of FWHM of the two Sn core level peaks of the fresh surface $(1.46 \mathrm{eV})$ is larger than what is expected from a single core level peak. In fact, after $30 \mathrm{~s}$ of sputtering, these two peaks can be seen to be composed of at least two peaks. As sputtering further proceeds, these two peaks become higher and narrower. These observations also indicate that the two Sn core level peaks also involve surface oxide components.

In the top spectrum of Fig. $6 \mathrm{a}$, the raw data of the two Te $3 \mathrm{~d}_{5 / 2}$ peaks with background subtracted are displayed together with three fitted peaks. The peak positioned at $576.1 \mathrm{eV}$ can be assigned to $\mathrm{TeO}_{2}$, and the peak at $572.2 \mathrm{eV}$ corresponds to the $\mathrm{Te}^{2-}$ state of SnTe, which agree with their previously reported standard values. ${ }^{45}$ It is worthwhile to note that the peak at
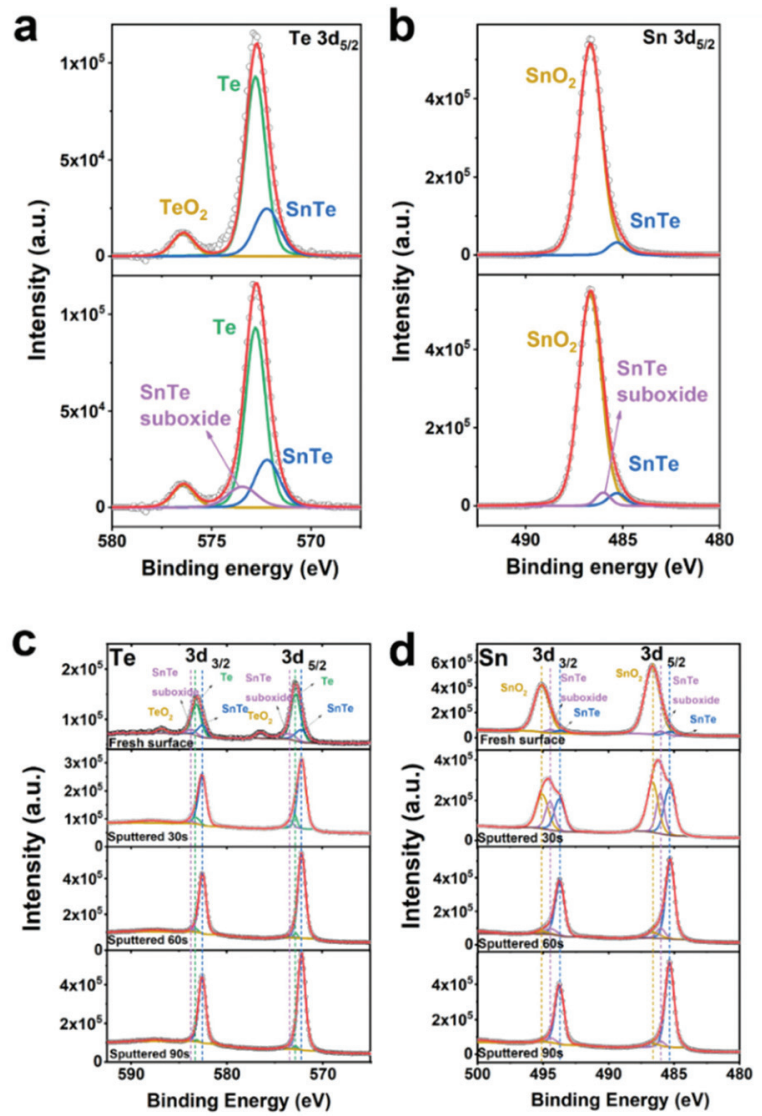

Fig. 6 X-ray photoelectron spectroscopy (XPS) spectra obtained from the surface of the SnTe (001) sample exposed to air after the MBE growth. (a) Te $3 d_{5 / 2}$ and (b) $S n 3 d_{5 / 2}$ with Shirley background subtraction; the top and bottom graphs display the fitting curves before and after adding an additional peak of the SnTe suboxide structure, respectively. (c) Te 3d and (d) Sn 3d, the four graphs from top to bottom show the spectra of the fresh surface, and those after sputtered for $30 \mathrm{~s}, 60 \mathrm{~s}$ and $90 \mathrm{~s}$, respectively, together with the fitted curves by including the peak of the SnTe suboxide structures.

$572.8 \mathrm{eV}$ in the top graph in Fig. 6a corresponds to the elemental $\mathrm{Te}^{0}$ state. ${ }^{45}$ Its existence can be explained by the mechanisms proposed by Neudachina et $a l .{ }^{45}$ that $\mathrm{Te}^{0}$ may be an intermediate for $\mathrm{Te}^{4+}\left(\mathrm{TeO}_{2}\right)$ formation: $\mathrm{Te}^{2-} \stackrel{v_{1}}{\longrightarrow} \mathrm{Te}^{0} \stackrel{\nu_{2}}{\longrightarrow} \mathrm{Te}^{4+}$. $\mathrm{Te}^{0}$ accumulated at the surface is attributed to the fact that the rate of the first step likely exceeds that of the second step $\left(v_{1}>\right.$ $v_{2}$ ). It has been reported that the Te peaks of $\mathrm{Te}^{4+}$ in $\mathrm{TeO}_{2}$, elemental $\mathrm{Te}^{0}, \mathrm{Te}^{2-}$ in SnTe have approximately symmetric shapes. ${ }^{46-50}$ In the top graph of Fig. $6 \mathrm{a}$, the raw data of the Te $3 \mathrm{~d}_{5 / 2}$ peaks with background subtracted are shown together with three symmetric fitted curves of $\mathrm{Te}^{4+}, \mathrm{Te}^{0}$ and $\mathrm{Te}^{2-}$; however, the overall fitted curve in red does not fit the raw data well. Obviously, an additional peak in the left shoulder of the $\mathrm{Te}^{0}$ peak must be added to obtain the best fit of the raw data as shown in the bottom graph of Fig. 6a. In the top graph of Fig. $6 \mathrm{~b}$, the raw data of the $\mathrm{Sn} 3 \mathrm{~d}_{5 / 2}$ peak with background subtracted are displayed together with two fitted peaks. The peak positioned at $486.7 \mathrm{eV}$ can be assigned to the $\mathrm{Sn}^{4+}$ state 
of $\mathrm{SnO}_{2}$, and the peak at $485.3 \mathrm{eV}$ corresponds to the $\mathrm{Sn}^{2+}$ state of SnTe, which agrees with their previously reported standard values. ${ }^{45}$ It has also been reported that the $\mathrm{Sn}^{4+}$ and $\mathrm{Sn}^{2+}$ peaks have symmetric shapes. ${ }^{51-54}$ In the top graph of Fig. 6b, the raw data of the $\mathrm{Sn} 3 \mathrm{~d}_{5 / 2}$ peak with background subtracted are shown together with two symmetric fitted curves of $\mathrm{Sn}^{4+}$ and $\mathrm{Sn}^{2+}$; however, the overall fitted curve in red does not fit the raw data well. Similarly, an additional peak is also needed to obtain a better fit as shown in the bottom graph of Fig. $6 \mathrm{~b}$. Based on this analysis, we have performed data fitting for all the original XPS spectra by adding a peak at the left shoulder of each of the elemental $\mathrm{Te}^{0}$ and $\mathrm{Sn}^{2+}$ peaks in SnTe. Indeed, all the raw data including those obtained after sputtering are now well fitted as shown in Fig. $6 \mathrm{c}$ and $\mathrm{d}$ with all the binding energies of the major peaks of $\mathrm{Te}^{4+}$ in $\mathrm{TeO}_{2}$, elemental $\mathrm{Te}^{0}$, $\mathrm{Te}^{2-}$ in SnTe, $\mathrm{Sn}^{4+}$ in $\mathrm{SnO}_{2}$, and $\mathrm{Sn}^{2+}$ in SnTe consistent with the reported standard values. ${ }^{32,45,50,55,56}$ Interestingly, previous XPS studies ${ }^{32,57}$ reported that the oxidized Te surface and SnTe surface each contain a small suboxide peak with binding energies similar to that of the additional peaks described in Fig. 6 . It is believed that the two additional peaks in our XPS spectra provide the evidence of the existence of the partially oxidized SnTe surface structures predicated from our theoretical studies.

As shown in the top second graph of Fig. 6c, after sputtering for $30 \mathrm{~s}$, the $\mathrm{TeO}_{2}$ peak becomes undetectable, while the peaks associated with the elemental $\mathrm{Te}^{0}$ decrease obviously, which are consistent with the mechanism mentioned above that elemental $\mathrm{Te}^{0}$ is an intermediate during the formation of $\mathrm{TeO}_{2}$. However, it should be noted that both the SnTe suboxide and SnTe peak increase. The bottom two graphs in Fig. 6c display that further sputtering results in a gradual decrease in the intensities of the SnTe suboxide and elemental $\mathrm{Te}^{0}$ peaks. As shown in the top second graph of Fig. 6d, after sputtering for $30 \mathrm{~s}$, the peak intensity of $\mathrm{SnO}_{2}$ decreases significantly and that of both the SnTe suboxide and SnTe increases obviously. The bottom two graphs in Fig. 6d show that both the $\mathrm{SnO}_{2}$ and $\mathrm{SnTe}$ suboxide peaks drop gradually upon further sputtering. It is important to mention that the SnTe suboxide peaks of Te and Sn core levels show higher intensities after the surface was sputtered for the first $30 \mathrm{~s}$ as mentioned above as compared to that of the fresh surface, which likely indicates that these SnTe suboxide structures are mainly located below the major oxides of $\mathrm{Te}^{0}, \mathrm{TeO}_{2}$ and $\mathrm{SnO}_{2}$. It is worthwhile to mention that the electrolyte used in our electrochemical measurements is a $0.5 \mathrm{M} \mathrm{H}_{2} \mathrm{SO}_{4}$ solution, which can etch away these major oxides, such that the SnTe surfaces with Sn vacancies and partially oxidized structures are exposed to make the $\mathrm{H}$ adsorption easier and enhance the HER activity.

\section{WAL effect on the SnTe (001), (111) and (211) samples}

The TSSs in TCI SnTe can be experimentally probed by lowtemperature magneto-transport measurements. The WAL effect due to spin-momentum locking and accumulation of the $\pi$ Berry phase in magneto-transport is considered as a characteristic feature of TSSs. In general, the transport of TSSs, as nontrivial two-dimensional (2D) states, usually occurs concurrently with that of the trivial $3 \mathrm{D}$ bulk states. ${ }^{58}$ However, TSSs lead to the 2D WAL effect in perpendicular applied magnetic field while 3D bulk states give rise to the 3D WAL effect, which is independent of the direction of the magnetic field. ${ }^{59}$ The WAL effect due to TSSs can be obtained by subtracting the WAL effect in parallel applied magnetic field from that in perpendicular applied magnetic field. As shown in Fig. 7a-c, the SnTe samples with the surface orientation of (001), (111) and (211) all exhibit sharp dips in the magnetoresistance $\left(\mathrm{MR}(\%)=\frac{R(B)-R(0)}{R(0)} \times 100 \%\right)$ around zero magnetic field when magnetic field is applied in both perpendicular and parallel directions. The sharp dip is a hallmark of the WAL effect due to quantum correction to the classical quadratic magnetoresistance. Fig. 7d-f show that after subtracting the $3 \mathrm{D}$ bulk contribution, the WAL effect due to TSSs in terms of magnetoconductance $\Delta G=\Delta G\left(B_{\perp}\right)-\Delta G\left(B_{\|}\right)$only remains in the SnTe (001) and SnTe (111) samples. No noticeable TSS-induced WAL feature for the SnTe (211) sample is observed. ${ }^{58}$ These results are consistent with the fact that TSSs protected by crystal symmetry exist and are robust against disorder in the highly symmetric surfaces of SnTe. ${ }^{8,9}$ The high HER performance of the SnTe (001) and SnTe (111) samples observed in this work is thus believed to be attributed to the TSSs. The absence or fragility of TSSs in the lowly symmetric SnTe (211) seemingly leads to the observed inferior catalytic activity.

We would like to point out that WAL effect is a quantum coherent transport at low temperature. We measured the WAL effect in SnTe (001) at different temperatures (as shown in Fig. $\mathrm{S} 29 \dagger$ ) and found that it gradually vanishes when the temperature increases up to $10 \mathrm{~K}$, which is quite close to the value reported by others for SnTe (111). ${ }^{58}$ No WAL effect is expected to be observed at room temperature because as temperature increases, inelastic electron-electron interaction and electronphonon scattering will be enhanced, which leads to phase decoherence and thus disappearance of the WAL effect. ${ }^{58,60}$

However, the disappearance of the WAL effect at higher temperature does not correspond to the non-existence of TSSs in SnTe. Chen et al. ${ }^{61}$ used high resolution angle-resolved photoemission spectroscopy (ARPES) to directly probe the TSSs of the $\mathrm{Bi}_{2} \mathrm{Te}_{3}$ and $\mathrm{Bi}_{2} \mathrm{Se}_{3}$ upon exposure to various environments. It was found that the topological order is robust even when the surface is exposed to the ambient environment at room temperature $(300 \mathrm{~K})$. Hoefer et al. ${ }^{48}$ also demonstrated that at room temperature, the characteristic linear dispersion of the surface states of $\mathrm{Bi}_{2} \mathrm{Te}_{3}$ was clearly observable in ARPES, suggesting the presence of the massless Dirac fermions. Thus, it is reasonable to speculate that the TSSs of SnTe also persist up to room temperature. It is also worthwhile to mention that the TSSs of a TCI are protected by a point-group symmetry of the crystal lattice rather than by TRS. ${ }^{9}$ Our HRXRD spectra (Fig. 1) confirm that all three SnTe samples maintain their rock-salt structure at room temperature together with the fact that the melting point of SnTe is $1060 \mathrm{~K}$; thus phase transition 

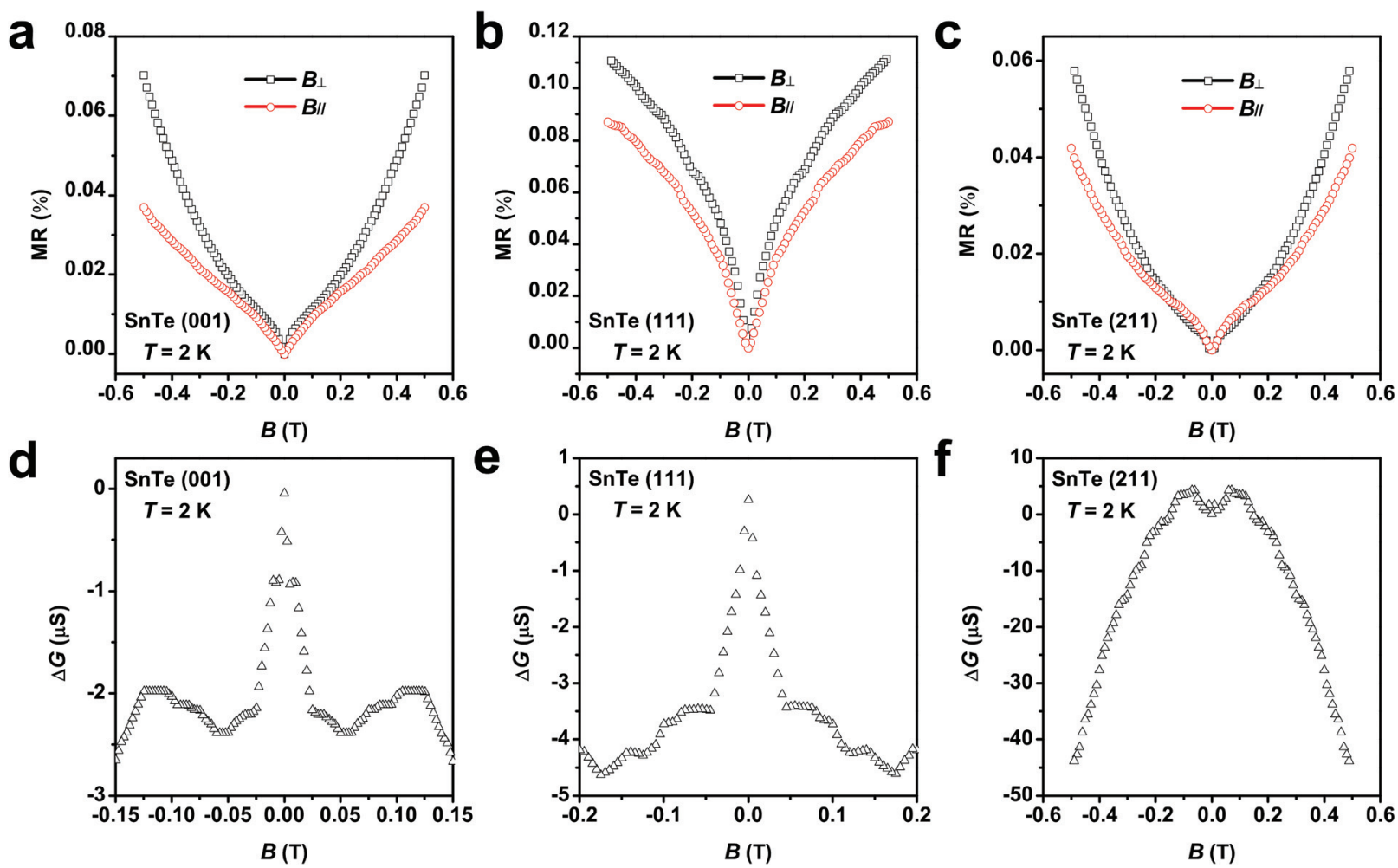

Fig. 7 WAL effect observed in the SnTe (001), SnTe (111) and SnTe (211) samples at 2 K. (a-c) Magnetoresistance in (a) SnTe (001), (b) SnTe (111) and (c) SnTe (211) under perpendicular $\left(B_{\perp}\right)$ and parallel $\left(B_{\|}\right)$magnetic field. (d-f) Magnetoconductance calculated by the subtraction $\Delta G\left(B_{\perp}\right)-\Delta G\left(B_{\|}\right)$as a function of magnetic field of (d) SnTe (001), (e) SnTe (111) and (f) SnTe (211).

or crystal symmetry breaking is not expected to occur at room temperature, which also supports the fact that the TSSs of the SnTe (001) and (111) could still exist at room temperature.

\section{Further discussion on the role of the TSSs}

In the previous sections, we have shown that even the SnTe (211) sample has the roughest surface; however, its HER performance is the lowest among the three SnTe samples studied in our work. Someone may argue that this observation may be simply due to the generation of various facets at these rough surfaces and they may have different intrinsic activities. Certainly, a rough surface could bring out facets with various orientations at the upper most surface of a sample. As shown in our recent work, ${ }^{5}$ among five $\mathrm{Bi}_{2} \mathrm{Te}_{3}(001)$ thin films with different thicknesses, it is likely that they also contain surface facets with various orientations, and the $48 \mathrm{~nm}$ thin film with the roughest surface enjoys the highest HER performance. This observation indeed could rule out the facet factor being a significant negative contribution while supporting that a rougher surface could have more active sites for the HER. For the three SnTe samples studied in this work, as described earlier regarding the TOF values, the dominating factor among the three SnTe samples in their HER performance is not the number of available active sites, instead it is about how active these sites are among the three samples, while their intrinsic activity is dominated by the TSS contribution. In fact it has a big contrast between the (211) oriented thin film and the other two thin films as shown in our theoretical calculations regarding their $\Delta G_{\mathrm{H}}$ values. In addition, the TOF values as shown in Fig. $3 \mathrm{~d}$ and Table 1 indicate that the intrinsic activities of the (001) and (111) samples could be 50 to 100 times higher than that of the (211) sample at a potential of $200 \mathrm{mV}$. It is well known that TSSs can float to the top of the sample surface and they are robust and can survive against the surface defects or non-full oxidation and as demonstrated by other researchers theoretically ${ }^{6}$ and by us experimentally. ${ }^{5,62}$ For the TCI SnTe, the TSSs come from the crystalline interface underlying the rough surface as protected by the mirror symmetry. Thus even though the (211) surface has the highest roughness corresponding to the highest number of active sites, however, these sites are much less active; therefore the positive contribution from the higher number of active sites is overwhelmed by the TSS effect that is only available and robust for the (001) and (111) orientations among the three orientations studied.

It has been previously reported that the high bulk conductivities of TIs mask the effect of TSSs (which is true for narrow band-gap TIs (such as $\mathrm{Bi}_{2} \mathrm{Te}_{3}$ ) or TCIs (such as $\mathrm{SnTe}$ )), so the contribution from TSSs can be considered as negligible for their conductivity. ${ }^{63}$ Furthermore, the electrical conductivities of SnTe (001), (211) and (111) are determined to be (1.07 \pm $0.03) \times 10^{6},(4.40 \pm 0.06) \times 10^{5}$ and $(2.67 \pm 0.73) \times 10^{5} \mathrm{~S} \mathrm{~m}^{-1}$ at room temperature, which shows that the electrical conductivity 
of SnTe (211) is not the lowest among the three samples. Our observation that SnTe (211) suffers from the worst HER performance among the three samples rules out the possibility that the electrical conductivity is the key factor for the high HER performance of both SnTe (001) and (111) samples. Based on our theoretical studies, the difference in the $\Delta G_{\mathrm{H}}$ governs the HER activity of the three SnTe samples.

\section{Methods}

\section{Sample preparation}

All the SnTe (001), (111) and (211) samples studied in this work were fabricated on $n+$ GaAs substrates using a VG-V80H MBE system equipped with in situ RHEED. Sample synthesis was conducted using a high-purity SnTe compound source. For the SnTe (001) sample, a SnTe layer was deposited directly on the GaAs (001) substrate at a substrate temperature of $22{ }^{\circ} \mathrm{C}$. For the SnTe (111) sample, a $5 \mathrm{~nm}$ ZnSe buffer layer was first deposited, followed by the growth of a $\mathrm{Bi}_{2} \mathrm{Te}_{3}:$ Fe layer at a substrate temperature of $242{ }^{\circ} \mathrm{C}$ (a lower temperature of $234^{\circ} \mathrm{C}$ was used for the first $5 \mathrm{~min}$, it was found that this twostep growth mode provided a better structural quality) and a SnTe layer at a substrate temperature of $222^{\circ} \mathrm{C}$. For the SnTe (211) sample, a $630 \mathrm{~nm}$ ZnSe buffer layer was deposited first, and then a $120 \mathrm{~nm}$ SnTe layer was deposited at a substrate temperature of $222{ }^{\circ} \mathrm{C}$. All the growth processes were performed in an ultrahigh-vacuum chamber with a basic pressure better than $1.0 \times 10^{-9}$ Torr.

\section{Material characterization}

All the SnTe (001), (111) and (211) thin film samples were characterized by HRXRD (PANalytical multipurpose diffractometer using $\mathrm{Cu} \mathrm{K} \alpha_{1}$ X-rays with a wavelength of $1.54056 \AA$ ), TEM (JEOL JEM-2010F with an acceleration voltage of $200 \mathrm{kV}$ ), and AFM (Dimension 3100 with a NanoScope IIIa controller (Digital Instruments) using the tapping mode). All the XPS measurements were performed using Kratos-Axis Ultra DLD XPS ex situ. This instrument was equipped with a monochromatic Al K $\alpha$ X-ray source (photon energy $1486.7 \mathrm{eV}, 150 \mathrm{~W}$ ), and the measurements were taken in hybrid lens mode with an energy step of $100 \mathrm{meV}$, a pass energy of $40 \mathrm{eV}$, and a large measuring area of $1 \times 2 \mathrm{~mm}^{2}$. The ion sputtering of the film was handled using Ar ions with $4 \mathrm{kV}, 3 \mathrm{~mm} \times 3 \mathrm{~mm}$ raster, and $140 \mu \mathrm{A}$ extractor current, and the sputtering rate was found to be similar to that of $\mathrm{SiO}_{2}, \sim 1 \AA \mathrm{s}^{-1}$.

\section{Electrochemical measurements for the HER}

All the electrochemical measurements were performed in a standard three-electrode electrolyzer connected to a $\mathrm{CHI} 660 \mathrm{E}$ electrochemical workstation ( $\mathrm{CH}$ Instruments), using SnTe (001), (111) or (211) as the working electrode, respectively, a graphite rod as a counter electrode, a standard $\mathrm{Ag} / \mathrm{AgCl}$ electrode (saturated $\mathrm{KCl}$ solution) as the reference electrode, and $0.5 \mathrm{M} \mathrm{H}_{2} \mathrm{SO}_{4}$ as the electrolyte (degassed by $\mathrm{N}_{2}$, purity $\sim 99.995 \%$ ). In constructing the working electrode, a piece of the as-grown sample is connected to a poly-ether-ether-ketone (PEEK) electrode via a conductive glassy carbon clip, one side of the clip touches the back side of the GaAs substrate and the other side connects a small part of the sample surface to the PEEK electrode. As mentioned in our previous work, ${ }^{5}$ the GaAs substrate has negligible HER activity. LSV was performed using a scan rate of $5 \mathrm{mV} \mathrm{s}^{-1}$. EIS measurements were carried out at open circuit potential over a frequency range of $10^{6}$ to $0.01 \mathrm{~Hz}$ with a perturbation voltage amplitude of $5 \mathrm{mV}$. The impedance data were fitted to a simplified Randles circuit to extract the series and charge-transfer resistances. All data presented were $i R$ corrected. The potential values shown were with respect to the reversible hydrogen electrode.

\section{Magneto-transport characterization}

Electrical contacts were prepared by thermal evaporation of $\mathrm{Cr} /$ $\mathrm{Au}$ thin films with typical thicknesses of $10 \mathrm{~nm} / 100 \mathrm{~nm}$ through shadow masks on SnTe (001), (111) and (211) thin films. The samples were subsequently patterned into fourterminal devices. The magneto-transport measurements were performed in a Quantum Design Physical Property Measurement System (PPMS, Quantum Design) equipped with a rotational sample holder.

\section{Theoretical calculations}

We conducted DFT calculations with plane-wave basis sets and the PBE exchange-correlation functional ${ }^{64}$ implemented in the Quantum Espresso package (v.6.1). ${ }^{65}$ We applied the Optimized Norm-Conserving Vanderbilt (ONCV) pseudopotentials $^{66-68}$ for $\mathrm{H}, \mathrm{Sn}$ and Te and ultrasoft pseudopotential $^{69}$ for $\mathrm{O}$, and the kinetic energy cutoff is 50 Ry. We did spin-orbit coupling (SOC) calculations with fully relativistic pseudopotentials to identify the gapless TSSs. An experimental lattice constant of $6.328 \AA$ was adopted for the SnTe rock salt structure. $^{30,70,71}$ In the structural optimization, the upper 2-monolayer (ML) with the adsorbed $\mathrm{H}$ atom could move until the force on each atom was smaller than 0.0001 Ry per Bohr, and the other atoms were fixed at the bulk positions. In electronic structure calculations, the surface structures without $\mathrm{H}$ adsorption were obtained by directly removing the $\mathrm{H}$ atom without any structural relaxation. With periodic boundary conditions, we kept at least $12 \AA$ vacuum to avoid interactions between neighboring replicas. We used the $6 \times 6 \times 1$ Monkhorst-Pack $k$-point mesh ${ }^{72}$ in the structural optimization of the $(1 \times 1)$ SnTe $(001)$ unit cell, and $8 \times 8 \times 1$ for electronic structure calculations with SOC. More detailed computationalmodel configurations are included in ESI note $3 . \dagger$

\section{Conclusions}

We have successfully synthesized high-quality SnTe (111), (001), and (211) samples on GaAs substrates by the MBE technique. Their structural properties were examined by RHEED, HRXRD, cross-sectional HRTEM, and AFM. Their performance in the HER was characterized by various electrochemical 
measurements. It was found that the SnTe (001) sample exhibits the lowest $\eta$, the lowest Tafel slope, and the lowest $R_{\text {ct }}$ among the three SnTe samples. As revealed by AFM analysis, the three surfaces show very different surface morphologies. Using the LSV and CV measurements, their intrinsic electrochemical activities were extracted with the results that SnTe (001) and (111) surfaces outperform the (211) surface. We have carried out DFT calculations to study the underlying mechanism of our experimental observations. We found that while the pure SnTe surfaces do not favor the $\mathrm{H}$ adsorption, the Sn vacancy or the partial oxidation in the surfaces lifts the Dirac cones and makes the $\mathrm{H}$ adsorption easier, which boosts the HER performance. The overall performance of the (001) and (111) surfaces with TSSs is better than that of the (211) surface without TSSs. These theoretical findings were further supported by XPS analysis and magneto-transport measurements. The issues about the effect of possible surface facets and the contrast in the activity of their active sites among the three samples were also addressed, which further confirm the important role of the TSS effect that is only available and robust for the (001) and (111) oriented samples. This work demonstrates that the TSSs and mirror symmetry of the SnTe (001) and (111) surfaces play an important role in their efficient HER performance and shed light on achieving cost-effective electrocatalysts in water splitting based on the use of TCIs.

\section{Author contributions}

Q.Q. and B.L. contributed equally to this work. Q.Q. and I.K.S. initiated this study and further designed the experiments; Q.Q. carried out the sample synthesis and conducted the structural characterization and electrochemical measurements; J.L. helped to do the analyses of RHEED patterns and TEM images. B.L. and D.P. carried out the theoretical calculations; H.T.L. and J.N.W conducted the magneto-transport measurements. Q.Q., I.K.S., D.P., B.L., H.T.L. and J.N.W wrote the manuscript. All authors performed the data analysis and discussions.

\section{Conflicts of interest}

There are no conflicts to declare.

\section{Acknowledgements}

This research was funded by the Research Grants Council of the Hong Kong Special Administrative Region, China, under Grant Numbers 16304515, 16301418, C6013-16E, 16308020, C6025-19G, C6011-20G and William Mong Institute of Nano Science and Technology under Project Number WMINST19SC06 and WMINST19SC07. D.P. acknowledges support from the Croucher Foundation through the Croucher Innovation Award and the Energy Institute at the Hong Kong University of Science and Technology.

\section{Notes and references}

1 C. R. Rajamathi, U. Gupta, N. Kumar, H. Yang, Y. Sun, V. Süß, C. Shekhar, M. Schmidt, H. Blumtritt, P. Werner, B. Yan, S. Parkin, C. Felser and C. N. R. Rao, Adv. Mater., 2017, 29, 1606202.

2 C. R. Rajamathi, U. Gupta, K. Pal, N. Kumar, H. Yang, Y. Sun, C. Shekhar, B. Yan, S. Parkin, U. V. Waghmare, C. Felser and C. N. R. Rao, ChemPhysChem, 2017, 18, 23222327.

3 J. Li, H. Ma, Q. Xie, S. Feng, S. Ullah, R. Li, J. Dong, D. Li, Y. Li and X.-Q. Chen, Sci. China Mater., 2018, 61, 23-29.

4 L. Li, J. Zeng, W. Qin, P. Cui and Z. Zhang, Nano Energy, 2019, 58, 40-46.

5 Q. Qu, B. Liu, J. Liang, H. Li, J. N. Wang, D. Pan and I. K. Sou, ACS Catal., 2020, 10, 2656-2666.

6 H. Chen, W. Zhu, D. Xiao and Z. Zhang, Phys. Rev. Lett., 2011, 107, 056804.

7 J. Xiao, L. Kou, C.-Y. Yam, T. Frauenheim and B. Yan, ACS Catal., 2015, 5, 7063-7067.

8 L. Fu, Phys. Rev. Lett., 2011, 106, 106802.

9 T. H. Hsieh, H. Lin, J. Liu, W. Duan, A. Bansil and L. Fu, Nat. Commun., 2012, 3, 982.

10 Y. Tanaka, Z. Ren, T. Sato, K. Nakayama, S. Souma, T. Takahashi, K. Segawa and Y. Ando, Nat. Phys., 2012, 8, 800-803.

11 J. Liu, W. Duan and L. Fu, Phys. Rev. B: Condens. Matter Mater. Phys., 2013, 88, 241303.

12 J. Wang, N. Wang, H. Huang and W. Duan, Chin. Phys. B, 2016, 25, 117313.

13 A. A. Taskin, F. Yang, S. Sasaki, K. Segawa and Y. Ando, Phys. Rev. B: Condens. Matter Mater. Phys., 2014, 89, 121302.

14 S. Ramesh, N. Kobayashi and Y. Horikoshi, J. Cryst. Growth, 1991, 111, 752-756.

15 K. Zhao, L. Ye, M. C. Tamargo and A. Shen, Phys. Status Solidi C, 2012, 9, 1809-1812.

16 Y. Gong, K. Zhu, Z. Li, Y. Zang, X. Feng, D. Zhang, C. Song, L. Wang, W. Li, X. Chen, X.-C. Ma, Q.-K. Xue, Y. Xu and K. He, Nano Res., 2018, 11, 6045-6050.

17 R. Ishikawa, T. Yamaguchi, Y. Ohtaki, R. Akiyama and S. Kuroda, J. Cryst. Growth, 2016, 453, 124-129.

18 J. G. N. Thomas, Trans. Faraday Soc., 1961, 57, 16031611.

19 J. K. Nørskov, T. Bligaard, A. Logadottir, J. Kitchin, J. G. Chen, S. Pandelov and U. Stimming, J. Electrochem. Soc., 2005, 152, J23-J26.

20 T. Wang, X. Wang, Y. Liu, J. Zheng and X. Li, Nano Energy, 2016, 22, 111-119.

21 J. Zhang, T. Wang, P. Liu, Z. Liao, S. Liu, X. Zhuang, M. Chen, E. Zschech and X. Feng, Nat. Commun., 2017, 8, 15437.

22 Y. Ou, W. Tian, L. Liu, Y. Zhang and P. Xiao, J. Mater. Chem. A, 2018, 6, 5217-5228.

23 K. Wang, X. She, S. Chen, H. Liu, D. Li, Y. Wang, H. Zhang, D. Yang and X. Yao, J. Mater. Chem. A, 2018, 6, 5560-5565. 
24 Z. Chen, D. Cummins, B. N. Reinecke, E. Clark, M. K. Sunkara and T. F. Jaramillo, Nano Lett., 2011, 11, 4168-4175.

25 J. Yang, K. Wang, J. Zhu, C. Zhang and T. Liu, ACS Appl. Mater. Interfaces, 2016, 8, 31702-31708.

26 Q. Tang and D.-e. Jiang, ACS Catal., 2016, 6, 4953-4961.

27 A. J. Medford, A. Vojvodic, J. S. Hummelshøj, J. Voss, F. Abild-Pedersen, F. Studt, T. Bligaard, A. Nilsson and J. K. Nørskov, J. Catal., 2015, 328, 36-42.

28 H. Zhang, Q. Pan, Z. Sun and C. Cheng, Nanoscale, 2019, 11, 11505-11512.

29 J. Zhu, Z.-C. Wang, H. Dai, Q. Wang, R. Yang, H. Yu, M. Liao, J. Zhang, W. Chen and Z. Wei, Nat. Commun., 2019, 10, 1-7.

30 D. Zhang, H. Baek, J. Ha, T. Zhang, J. Wyrick, A. V. Davydov, Y. Kuk and J. A. Stroscio, Phys. Rev. B: Condens. Matter Mater. Phys., 2014, 89, 245445.

31 N. Berchenko, T. Story, M. Trzyna, S. Fadeev, K. Kurbanov, S. Adamiak, W. Bochnowski, A. Dziedzic and J. Cebulski, Surf. Interface Anal., 2016, 48, 547-551.

32 N. Berchenko, R. Vitchev, M. Trzyna, R. WojnarowskaNowak, A. Szczerbakow, A. Badyla, J. Cebulski and T. Story, Appl. Surf. Sci., 2018, 452, 134-140.

33 S. Ma, C. Guo, C. Xiao, F. Wu, M. Smidman, Y. Lu, H. Yuan and H. Wu, Adv. Funct. Mater., 2018, 28, 1803188.

34 P. O. Löwdin, J. Chem. Phys., 1950, 18, 365-375.

35 Y. Tanaka, T. Shoman, K. Nakayama, S. Souma, T. Sato, T. Takahashi, M. Novak, K. Segawa and Y. Ando, Phys. Rev. B: Condens. Matter Mater. Phys., 2013, 88, 235126.

36 Z. Fang, E. Wang, Y. Chen, X. Hou, K.-C. Chou, W. Yang, J. Chen and M. Shang, ACS Appl. Mater. Interfaces, 2018, 10, 30811-30818.

37 H. Huang, X. Fan, D. J. Singh and W. Zheng, ACS Omega, 2018, 3, 10058-10065.

38 N. Ostojic, Z. Duan, A. Galyamova, G. Henkelman and R. M. Crooks, J. Am. Chem. Soc., 2018, 140, 13775-13785.

39 J. Xiao and T. Frauenheim, J. Phys. Chem. Lett., 2012, 3, 2638-2642.

40 H. Li, C. Tsai, A. L. Koh, L. Cai, A. W. Contryman, A. H. Fragapane, J. Zhao, H. S. Han, H. C. Manoharan and F. Abild-Pedersen, Nat. Mater., 2016, 15, 48-53.

41 Y. Yin, J. Han, Y. Zhang, X. Zhang, P. Xu, Q. Yuan, L. Samad, X. Wang, Y. Wang and Z. Zhang, J. Am. Chem. Soc., 2016, 138, 7965-7972.

42 K. Chi, X. Tian, Q. Wang, Z. Zhang, X. Zhang, Y. Zhang, F. Jing, Q. Lv, W. Yao and F. Xiao, J. Catal., 2020, 381, 4452.

43 Y. Yan, J. Lin, J. Cao, S. Guo, X. Zheng, J. Feng and J. Qi, J. Mater. Chem. A, 2019, 7, 24486-24492.

44 M. Bala, A. Masarrat, V. Kumar, S. Ojha, K. Asokan and S. Annapoorni, J. Appl. Phys., 2020, 127, 245108.

45 V. S. Neudachina, T. B. Shatalova, V. I. Shtanov, L. V. Yashina, T. S. Zyubina, M. E. Tamm and S. P. Kobeleva, Surf. Sci., 2005, 584, 77-82.

46 A. Mekki, G. D. Khattak and L. E. Wenger, J. Non-Cryst. Solids, 2006, 352, 3326-3331.
47 A. Mekki, G. D. Khattak and L. E. Wenger, J. Non-Cryst. Solids, 2005, 351, 2493-2500.

48 K. Hoefer, C. Becker, D. Rata, J. Swanson, P. Thalmeier and L. H. Tjeng, Proc. Natl. Acad. Sci. U. S. A., 2014, 111, 14979.

49 C. I. Fornari, P. H. O. Rappl, S. L. Morelhão, T. R. F. Peixoto, H. Bentmann, F. Reinert and E. Abramof, APL Mater., 2016, 4, 106107.

50 Thermo Fisher Scientific Inc, https://xpssimplified.com/ elements/tellurium.php. 2013-2020.

51 J. K. Kwak, K. H. Park, D. Y. Yun, D. U. Lee, T. Kim, D. I. Son, J. H. Han and J. Y. Lee, J. Korean Phys. Soc., 2010, 57, 1803-1806.

52 Q. Jiang, L. Zhang, H. Wang, X. Yang, J. Meng, H. Liu, Z. Yin, J. Wu, X. Zhang and J. You, Nat. Energy, 2016, 2, 16177.

53 R. G. Motsoeneng, I. Kortidis, S. S. Ray and D. E. Motaung, ACS Omega, 2019, 4, 13696-13709.

54 S. Lu, X. Hu, H. Zheng, J. Qiu, R. Tian, W. Quan, X. Min, P. Ji, Y. Hu, S. Cheng, W. Du, X. Chen, B. Cui, X. Wang and W. Zhang, Sensors, 2019, 19, 2958.

55 J. F. Moulder and J. Chastain, Handbook of X-ray Photoelectron Spectroscopy: A Reference Book of Standard Spectra for Identification and Interpretation of XPS Data, Physical Electronics Division, Perkin-Elmer Corporation, 1992.

56 ThermoFisherScientificInc. https:/xpssimplified.com/ elements/tin.php. 2013-2020.

57 T. Kosmala, H. Coy Diaz, H.-P. Komsa, Y. Ma, A. V. Krasheninnikov, M. Batzill and S. Agnoli, Adv. Energy Mater., 2018, 8, 1800031.

58 R. Akiyama, K. Fujisawa, T. Yamaguchi, R. Ishikawa and S. Kuroda, Nano Res., 2016, 9, 490-498.

59 H.-T. He, G. Wang, T. Zhang, I.-K. Sou, G. K. L. Wong, J.-N. Wang, H.-Z. Lu, S.-Q. Shen and F.-C. Zhang, Phys. Rev. Lett., 2011, 106, 166805.

60 B. L. Altshuller, A. G. Aronov and D. E. Khmelnitsky, Solid State Commun., 1981, 39, 619.

61 C. Chen, S. He, H. Weng, W. Zhang, L. Zhao, H. Liu, X. Jia, D. Mou, S. Liu, J. He, Y. Peng, Y. Feng, Z. Xie, G. Liu, X. Dong, J. Zhang, X. Wang, Q. Peng, Z. Wang, S. Zhang, F. Yang, C. Chen, Z. Xu, X. Dai, Z. Fang and X. J. Zhou, Proc. Natl. Acad. Sci. U. S. A., 2012, 109, 36943698.

62 Q. L. He, Y. H. Lai, Y. Lu, K. T. Law and I. K. Sou, Sci. Rep., 2013, 3, 2497.

63 S. Cai, J. Guo, V. A. Sidorov, Y. Zhou, H. Wang, G. Lin, X. Li, Y. Li, K. Yang, A. Li, Q. Wu, J. Hu, S. K. Kushwaha, R. J. Cava and L. Sun, npj Quantum Mater., 2018, 3, 62.

64 J. P. Perdew, K. Burke and M. Ernzerhof, Phys. Rev. Lett., 1996, 77, 3865.

65 P. Giannozzi, O. Andreussi, T. Brumme, O. Bunau, M. B. Nardelli, M. Calandra, R. Car, C. Cavazzoni, D. Ceresoli and M. Cococcioni, J. Phys.: Condens. Matter, 2017, 29, 465901.

66 P. Scherpelz, M. Govoni, I. Hamada and G. Galli, J. Chem. Theory Comput., 2016, 12, 3523-3544. 
67 M. Schlipf and F. Gygi, Comput. Phys. Commun., 2015, 196, 36-44.

68 D. R. Hamann, Phys. Rev. B: Condens. Matter Mater. Phys., 2013, 88, 085117.

69 D. Vanderbilt, Phys. Rev. B: Condens. Matter Mater. Phys., 1990, 41, 7892.
70 J. Wang, J. Liu, Y. Xu, J. Wu, B.-L. Gu and W. Duan, Phys. Rev. B: Condens. Matter Mater. Phys., 2014, 89, 125308.

71 Y.-X. Chen, F. Li, D. Li, Z. Zheng, J. Luo and P. Fan, Materials, 2019, 12, 3001.

72 H. J. Monkhorst and J. D. Pack, Phys. Rev. B: Solid State, 1976, 13, 5188. 